\title{
Using Natural Language Processing and VetCompass to understand antimicrobial usage patterns in Australia
}

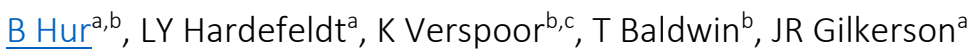

${ }^{a}$ Asia-Pacific Centre for Animal Health, Melbourne Veterinary School, University of Melbourne

${ }^{b}$ School of Computing and Information Systems, University of Melbourne

'Health and Biomedical Informatics Centre, University of Melbourne

\section{Abstract}

Currently there is an incomplete understanding of antimicrobial usage patterns in veterinary clinics in Australia. Knowledge of antimicrobial usage patterns are critical for the successful implementation and monitoring of antimicrobial stewardship programs. VetCompass Australia ${ }^{1}$ collects medical records from 181 clinics in Australia (as of May 2018). These records contain detailed information from individual consultations regarding the medications dispensed in the form of unstructured medical text. One unique aspect of VetCompass Australia is its focus on applying Natural Language Processing (NLP) and machine learning techniques to analyse the records, similar to efforts conducted in other medical studies².

The free text fields of 4,394,493 veterinary consultation records of dogs and cats between 2013 and 2018 were collated by VetCompass Australia and NLP techniques applied to enable the querying of the antimicrobial usage within these consultations. The NLP algorithms developed matched antimicrobial in clinical records with $96.7 \%$ Accuracy and an F1 Score of 0.85, as evaluated relative to expert annotations.

\section{Introduction}

Antimicrobial resistance (AMR) is a global health emergency ${ }^{3}$ and companion animals with bacteria containing AMR genes have been implicated as a potential reservoir of AMR genes that can affect human health ${ }^{4}$. Antimicrobial stewardship (AMS) is an effective way to reduce inappropriate antimicrobial usage ${ }^{5}$ but requires the ability to monitor antimicrobial usage patterns to measure their effectiveness.

The VetCompass database represents the largest corpus of veterinary records available for academic research and enables large scale analysis of Veterinary Medicine. Insights from analysis of big data can help to develop an evidence base to support clinical decisions and create more effective policies to drive better outcomes ${ }^{6}$.

Notes, written prescriptions, label text and inventory items dispensed were successfully extracted for each consultation from the VetCompass dataset. The data is, however, unstructured and accurate antimicrobial usage patterns cannot be directly queried in this format. The transformation of this to a structured dataset enables the use of VetCompass data to be used to describe what is being prescribed, and how often, in veterinary clinics in Australia.

Automatic text analysis can overcome the challenges of manual labelling of such data, enabling largescale extraction of key antimicrobial usage information in a structured format to allow subsequent analysis.

\section{Methods}

Clinical records from 4,394,493 consultations of dogs and cats were extracted from VetCompass Australia. 24,356 distinct inventory items which map to all prescriptions and consultation texts were 
extracted from these records. A subset of 2,346 of these items isolated to the clinics in the state of Victoria were examined by two veterinarians as a representative sample. The medication used and the presence of an antimicrobial as written in the inventory item was identified by the veterinarians, and annotated in situ. This was used to assess the accuracy of automatic methods for extracting this information from the item.

As there is human error in any annotation, the estimated overall error rate of annotations was performed by measuring the inter-rater agreement of a subset of 200 items that were labelled as either being an antimicrobial or not, in addition to identifying the medication name. The results of the two annotators were compared and the inter-rater agreement calculated using the Fleiss Kappa calculation?

A list of antimicrobials products was created, and this list was mapped to the individual antimicrobial ingredients.

The 24,356 distinct inventory items map to all 4,394,493 prescriptions and consultation texts, so the distinct inventory items were the only text requiring labelling.

A rule-based method was developed. This consisted of taking the free text of the distinct inventory items and list of antimicrobial products were broken down into individual word components (tokens). The first two tokens from VetCompass inventory items and the created list were compared and matched between each other to create a dictionary of all the tokens in each list with the number of character changes required for each token to match each other (Levenshtein Distance or edit distance $)^{8}$. Tokens that match completely have a value of 0 , whereas one-character difference is 1 , two-character difference is 2 , etc. The edit distance was then divided by the length of the token stored. Any item token that matched a medication or ingredient with a ratio of less than 0.25 was marked as a match. Another set of rules to make exceptions to this rule for brand names and to account for other exceptions (such as the chemical names such as 'Sodium' which is not a definitive medication name but which may match and create false positives, were also created.

Various machine learning methods, including eXtreme gradient boosting (XGBoost) ${ }^{9}$, Logistic Regression, Random Forest, Conditional Random Field (CRF), and Artificial Neural Networks (ANN) were also tested.

The rule-based and machine learning methods were trained and tested using the holdout method ${ }^{10}$. This consisted of splitting the 2,346 annotated items into two distinct sets. 1,846 for training and 500 held-out for testing the accuracy of the algorithms.

The antimicrobial agents annotated were also mapped to their importance rating in human medicine using the Australian Strategic Technical Advisory Groups (ASTAG) importance rating chart ${ }^{11}$.

To de-identify the clinical records, each clinic was assigned a number. To allow for geographical analysis of the medications dispensed, the names of the were mapped to postal codes. Specifically, clinic names associated with each consultation were extracted from VetCompass; and the Google Places Application Programming Interface (API) was used to identify the postal code.

The remaining list of distinct inventory items from the entire corpora containing 24,356 items was then annotated using the rule-based algorithm. This annotated list of distinct inventory items with labels was then mapped to the dataset of all the items dispensed in the 4,394,493 consultations.

\section{Results}


There was good inter-rater agreement between the two veterinarians (Fleiss Kappa $=0.868$ ) and the gold standard annotations were estimated at 95\% accuracy.

The final structured dataset for analysis consisted of 4,394,493 veterinary clinical consultations with labelled data of the antimicrobials dispensed and was interrogated using NLP methods. After testing both the rule-based algorithm and several machine learning algorithms, we found that the rule-based algorithm was the most effective. This algorithm identified antimicrobial agents with an accuracy of 96.7\% accuracy and an F1 score of 0.85 . These labels also include the ASTAG Rating of the antimicrobial agents and the geographical location of the clinic where the consultation was undertaken.

\section{Discussion}

The accuracy of the algorithm was in some ways limited by the inter-rater agreement. The algorithm's final performance was $96.37 \%$ when compared to the annotations created, but the interrater agreement of the annotations had an estimated accuracy of $95 \%$. This reflects the inconsistencies and errors in the annotations. These errors and inconsistencies make it unlikely to achieve significant performance improvement without more accurate annotations. This is similar to other NLP tasks limited by the accuracy of annotations ${ }^{12}$.

Of the best performing Machine learning models tested, XGBoost had a 91.9\% accuracy. The ANN using an ELMo-BiLSTM-CNN-CRF architecture ${ }^{13,14}$ had an accuracy of $94.4 \%$.

The methods used were only applied to the VetCompass corpus for companion animals. However, results could be expanded to other species and applied to other datasets within VetCompass.

Additionally, while a reliable corpus of antimicrobial ingredients has been mapped, further work is required to perform data analysis to describe the use patterns across Australia and compare against antimicrobial usage reports ${ }^{15}$.

\section{Conclusion}

In this paper we have taken 4,394,493 consult records from VetCompass and created a mapping of these consults to a structured dataset of antimicrobial agents given during these consults through the creation of custom algorithms at an accuracy of a $96.7 \%$ and an F1 Score of 0.85 when compared to expert annotations. This dataset can be readily queried to demonstrate the antimicrobial usage patterns of companion animal practices throughout Australia.

\section{References:}

1. McGreevy P, Thomson P, Dhand NK et al. VetCompass Australia: A National Big Data Collection System for Veterinary Science. 2017;15.

2. Murff HJ, FitzHenry F, Matheny ME et al. Automated Identification of Postoperative Complications Within an Electronic Medical Record Using Natural Language Processing. JAMA 2011;306:848-855.

3. Roca I, Akova M, Baquero F et al. The global threat of antimicrobial resistance: science for intervention. New Microbes New Infect 2015;6:22-29.

4. Allen HK, Donato J, Wang HH et al. Call of the wild: antibiotic resistance genes in natural environments. Nat Rev Microbiol 2010;8:251-259. 
5. Cisneros JM, Neth O, Gil-Navarro MV et al. Global impact of an educational antimicrobial stewardship programme on prescribing practice in a tertiary hospital centre. Clin Microbiol Infect 2014;20:82-88.

6. Martin-Sanchez F, Verspoor K. Big Data in Medicine Is Driving Big Changes: IMIA Yearb 2014;9:14-20.

7. Fleiss JL. Measuring nominal scale agreement among many raters. Psychol Bull 1971;76:378-382.

8. Levenshtein VI. Binary Codes Capable of Correcting Deletions, Insertions and Reversals. Sov Phys Dokl 1966;10:707.

9. Chen T, Guestrin C. XGBoost: A Scalable Tree Boosting System. Proceedings of the 22nd ACM SIGKDD International Conference on Knowledge Discovery and Data Mining - KDD '16. ACM Press, San Francisco, California, USA, 2016:785-794. http://dl.acm.org/citation.cfm?doid=2939672.2939785.

10. Kohavi R. A Study of Cross-validation and Bootstrap for Accuracy Estimation and Model Selection. Proceedings of the 14th International Joint Conference on Artificial Intelligence - Volume 2. Morgan Kaufmann Publishers Inc., San Francisco, CA, USA, 1995:1137-1143. http://dl.acm.org/citation.cfm?id=1643031.1643047.

11. Importance Ratings and Summary of Antibacterial Uses in Human and Animal Health in Australia. Antimicrob Resist 2018. https://www.amr.gov.au/resources/importanceratings-and-summary-antibacterial-uses-human-and-animal-health-australia. Retrieved December 112018.

12. Manning CD. Part-of-Speech Tagging from 97\% to 100\%: Is It Time for Some Linguistics? In: Gelbukh AF, editor. Computational Linguistics and Intelligent Text Processing. Springer Berlin Heidelberg, Berlin, Heidelberg, 2011:171-189.

http://link.springer.com/10.1007/978-3-642-19400-9_14.

13. Peters ME, Neumann $M$, lyyer $M$ et al. Deep contextualized word representations. ArXiv180205365 Cs 2018; http://arxiv.org/abs/1802.05365.

14. Reimers N, Gurevych I. Reporting Score Distributions Makes a Difference: Performance Study of LSTM-networks for Sequence Tagging. ArXiv170709861 Cs Stat 2017; http://arxiv.org/abs/1707.09861.

15. Hardefeldt LY, Holloway S, Trott DJ et al. Antimicrobial Prescribing in Dogs and Cats in Australia: Results of the Australasian Infectious Disease Advisory Panel Survey. J Vet Intern Med 2017;31:1100-1107. 


\section{University Library}

\section{- M M N E R VA A gateway to Melbourne's research publications}

Minerva Access is the Institutional Repository of The University of Melbourne

Author/s:

Hur, B;Hardefeldt, LY;Verspoor, K;Baldwin, T;Gilkerson, JR

Title:

Using natural language processing and VetCompass to understand antimicrobial usage patterns in Australia

Date:

2019-08-01

Citation:

Hur, B., Hardefeldt, L. Y., Verspoor, K., Baldwin, T. \& Gilkerson, J. R. (2019). Using natural language processing and VetCompass to understand antimicrobial usage patterns in Australia. Australian Veterinary Journal, 97, (8), pp.298-300. Wiley. https://doi.org/10.1111/ avj.12836.

Persistent Link:

http://hdl.handle.net/11343/255409 